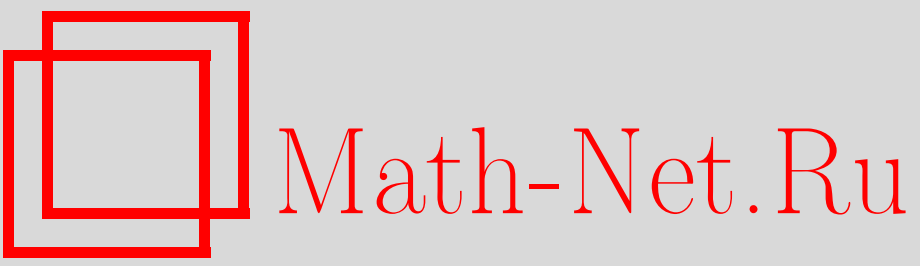

М. И. Штогрин, Непримитивные полициклы и гелицены, УМН, 2000, том 55, выпуск 2, 155-156

DOI: https://doi.org/10.4213/rm285

Использование Общероссийского математического портала Math-Net.Ru подразумевает, что вы прочитали и согласны с пользовательским соглашением

http://www.mathnet.ru/rus/agreement

Параметры загрузки:

IP : 54.80 .97 .219

26 апреля 2023 г., 11:34:03 


\title{
НЕПРИМИТИВНЫЕ ПОЛИЦИКЛЫ И ГЕЛИЦЕНЫ
}

\author{
М.И. Штогрин
}

Пусть $G$-конечный или бесконечный граф.. Мы полагаем, что бесконечньй граф имеет счетное число вершин, в каждой вершине сходится конечное число ребер, причем все эти числа ограничены в совокупности. Кроме того, любая конечная область на плоскости содержит конечное число вершин бесконечного плоского графа, непустое пересечение двух ребер всегда есть вершина, бесконечных вершин нет. Области, определяемые конечным или бесконечным плоским графом, назовем его гранями, причем ограниченную область назовем внутренней гранью, а неограниченную область назовем внешней гранью. В работах [1]-[3] определены примитивные полициклы.

ОПРЕДЕЛЕнИЕ. Конечный или бесконечньй плоский граф̆ $G$ вместе со всеми его внутренними гранями назовем непримитивным поли-r-циклом и обозначим его через $\Pi(G)$, если

(1) граф $G$ неразделим (при удалении любой его вершины $v$ граф $G-v$ остается связным);

(2) все внутренние грани граффа ограничены простыми циклами одной и той же длины $r$;

(3) пересечение любых двух внутренних граней есть одно ребро, одна вершина или пусто;

(4) число внутренних граней, сходящихся во внутренней вершине полицикла, равно $q, q \geqslant 4$;

(5) число внутренних граней, сходящихся в граничной вершине полицикла, меньше $q, q \geqslant 4$.

Внутреннюю грань плоского графа $G$ будем назьвать гранью поли- $r$-цикла $\Pi(G)$. Если в случае примитивного полицикла непустое пересечение двух граней всегда есть ребро, то в случае непримитивного полицикла это может быть либо ребро, либо вершина. Более того, у непримитивного полицикла всегда имеются хотя бы две грани, пересечение которых есть вершина. В противном случае в условиях (4), (5) должно фигурировать $q=3$, см. [3].

Пусть полицикл содержит не менее двух граней и не имеет внутренних вершин. Тогда подсчитаем число граней, сходяшихся в каждой граничной вершине полицикла. Все эти числа ограничены в совокупности. Возьмем максимум такого числа и прибавим к нему 1 . Полученное число обозначим через $q$. Если выполнены условия (1)-(3) и (5), то при $q=3$ полицикл является примитивным, а при $q \geqslant 4$ - непримитивным. Примитивные полициклы более удобно называть поли- $r^{3}$-циклами, а непримитивные - поли- $r^{q}$-циклами, $q \geqslant 4$.

Поли- $r^{q}$-цикл $\Pi(G), q \geqslant 4$, лежит в плоскости и представляет собою односвязную область. В самом деле, если бы в полицикле была дыра, ее окружал бы некоторый простой цикл из графа $G$. По теореме Жордана простой цикл в плоскости ограничивает некоторую конечную область. По соглашению об укладке на плоскости в конечной области содержится конечное число вершин (и ребер) бесконечного плоского графа. Поэтому область представляет собою объединение конечного числа $(\geqslant 1)$ внутренних граней. Никаких дыр в ней нет.

ТеОрема 1. Если (бес) конечный планарный граф $G$ допускает укладку на плоскости, реализующую поли-г-цикл П $(G)$, определяемый условиями (1)-(5), то лишь одну.

Точнее [1], реберный остов октаэдра или икосаэдра имеет соответственно 8 или 20 изоморфных реализаций в виде непримитивного поли- $3^{q}$-цикла, $q=4,5$. Реализация любого иного непримитивного поли- $r{ }^{q}$-цикла, $q \geqslant 4$, единственна в силу следствия из леммы. В самом деле, в силу условия (2) грани поли- $r^{q}$-цикла $\Pi(G)$ суть $r$-угольники. В силу следствия из леммы грани ограничены кратчайшими реберными циклами из графа $G$ длины $r$. Поэтому комбинаторное устройство полицикла $\Pi(G)$ однозначно определяется графом $G$.

Каждое ребро планарного графа, совпадающего с реберным остовом октаэдра или икосаэдра, принадлежит двум 3-циклам. Натянем на каждый 3-цикл грањь. Получим многогранник, гомеоморфный сфере. После выбрасывания одной грани получим полиэдр с краем, изоморфный полициклу, краем которого является край выкинутой грани, т.е. 3-цикл.

КРИТЕРИЙ. Отличный от реберного остова октаэдра и икосаэдра конечный планарный граф $G$ допускает на плоскости реализацию непримитивного поли-г ${ }^{q}$-цикла $\Pi(G)$, как всей конечной части плоской карты с условиями (1)-(5), если и только если

(а) каждое ребро принадлежит одному или двум простым циклам кратчайшей длиHol $r$

Работа выполнена при поддержке Российского фонда фундаментальных исследований (грант № 99-01-00010). 
(b) те ребра, каждое из которых принадлежст одному г-циклу, составляют простой чикл;

(c) пересечение любъх двух г-чиклов может бъть одно ребро, одна вершина или nyсто;

(d) число всех вместе взятых вершин и $r$-циклов графа превышает число ребер ровно на 1 ;

(е) все сходящиеся в одной вериине $r$-иикль составляют одну последовательность, в которой любье два соседние г-цикла имеют общее ребро (с концом в данной вериине);

(f) если последовательность из условия (e) замкнута, то число г-циклов в ней равно $q, \geqslant 4$

(g) если последовательность в условии (е) разомкнута, то число ее $r$-циклов меньue $q$.

Эквивалентность наборов условий из определения и критерия проверяется точно так же, как и в работе [2]. Следует только отметить, что условие (е) отметает появление псевдомногообразия (вместо нужного нам многообразия с краем) при натягивании граней на все $r$-циклы граба $G$ и что при выполнении условия (e) условия (f) и (g) совпадают с (4) и (5).

Пусть $\left(r^{q}\right)$ - разбиение сферы $\mathbb{S}^{2}$ при $r=3, q=3,4,5 ; r=4, q=3 ; r=5, q=3$, евклидовой плоскости $\mathbb{R}^{2}$ при $r=3, q=6 ; r=4, q=4 ; r=6, q=3$, гиперболической плоскости $\mathbb{H}^{2}$ при $r=3, q \geqslant 7 ; r=4, q \geqslant 5 ; r=5, q \geqslant 4 ; r=6, q \geqslant 4 ; r \geqslant 7, q \geqslant 3$ на правильные $r$-угольники с углами $2 \pi / q$ при вершинах. Как и в случае $q=3$, см. [1], имеет место

ПРЕДЛОЖЕНИЕ. Край $r$-угольника является кратчайшим циклом в разбиении $\left(r^{q}\right)$.

Каждому правильному разбиению $\left(r^{q}\right)$ или поли- $r^{q}$-циклу $\Pi(G)$ однозначно сопоставим комбинаторно изоморфный ему абстрактный двумерный полиэдр $\mathbf{K}\left(r^{q}\right)$ или $\mathbf{P}(G)$ соответственно, составленный из правильных евклидовых $r$-угольников, равных между собой.

Назовем поли- $r^{q}$-цикл $\Pi(G)$ собственным, если граф $G$ является частичным подграфом реберного остова правильного разбиения $\left(r^{q}\right)$, и несобственным в противном случае. Несобственный поли- $r^{q}$-цикл $\Pi(G)$ назовем гелиценом над $\left(r^{q}\right)$, если существует комбинаторное отображение $\Pi(G) \rightarrow\left(r^{q}\right)$, при котором не менее двух вершин имеют один и тот же образ.

Лемма. Пусть П $(G)$ - произвольный (бес)конечный непримитивный поли-r ${ }^{q}$-цикл, $r \geqslant 3, q \geqslant 4$. Тогда существует непрерывное локально изометрическое отображсение $f: \mathbf{P}(G) \rightarrow \mathbf{K}\left(r^{q}\right)$, сохраняющее клеточную структуру полиэдра $\mathbf{P}(G) \sim \mathbf{\Pi}(G)$.

Если $f$ является топологическим отображением полиэдра $\mathbf{P}(G)$ на его образ в $\mathbf{K}\left(r^{q}\right)$, то полицикл $\mathbf{\Pi}(G)$ собственный. Если отображение $f$ полиэдра $\mathbf{P}(G)$ на его образ в $\mathbf{K}\left(r^{q}\right)$ является не топологическим отображением, а гомоморфизмом, то полицикл $\Pi(G)$ является гелиценом над $\left(r^{q}\right)$. Эти два варианта исчерпывают все возможности для отображения $f$.

ТЕОрема 2. Любой несобственный (бес)конечный непримитивный поли-r ${ }^{q}-u и к л ~$ $\Pi(G), q \geqslant 4$, является гелиценом над $\left(r^{q}\right)$. Комбинаторное отображсение $\Pi(G)$ в $\left(r^{q}\right)$ единственно.

СлЕДСТВИЕ Из ЛЕммы. $В$ любом (бес)конечном непримитивном поли-r ${ }^{q}$-иикле $\Pi(G), r \geqslant 3, q \geqslant 4$, произвольный $r$-цикл графа $G$ ограничивает грань полицикла.

Если все вершины поли- $r^{q}$-цикла $\Pi(G)$ являются внутренними его вершинами, то $\Pi(G)=$ $\Pi\left(r^{q}\right) \sim \mathbf{P}\left(r^{q}\right)=\mathbf{K}\left(r^{q}\right) \sim\left(r^{q}\right)$, где $q \geqslant 6$ при $r=3 ; q \geqslant 4$ при $r=4$ или $r=5 ; q \geqslant 3$ при $r \geqslant 6$.

\section{СПИСОК ЛИТЕРАТУРЫ}

[1] Деза М., Штогрин М. И. // УМН. 1999. Т. 54. №6. С. 159-160. [2] Штогрин М. И. // УМН. 1999. Т. 54. № 6. С. 177-178. [3] Деза М., Штогрин М. И. // УМН. 2000. Т. 55. № 1. C. $179-180$.

LIENS, Ecole Normale Supérieure and CNRS, Paris; Математический институт им. В.А. Стеклова РАН
Принято редколлегией 26.01.2000 